\title{
The effectiveness of memory rehabilitation following neurological disabilities: A qualitative inquiry of patient perspectives
}

\author{
Roshan das Nair and Nadina B Lincoln \\ Institute of Work, Health and Organisations, University of Nottingham, \\ Nottingham, UK
}

The evidence for the effectiveness of memory rehabilitation following neurological conditions, mainly studied through quantitative methodologies, has been equivocal. This study aimed to examine feedback from participants who had been through a randomised controlled trial (RCT) comparing two types of memory rehabilitation with a self-help control. It was envisaged that this information would offer a detailed understanding of patient experience of going through a trial and the perceived effects of having attended group sessions. Through 31 in-depth interviews, data collected were thematically analysed. The seven themes identified highlighted improvements in insight and awareness of memory problems and their neurological conditions, knowledge and skills about using memory aids; and as a consequence, improvements in cognitive functions, mood, and confidence, assertiveness and control over their condition. Participants also reported an altered perspective of life that helped them deal with their problems, and the therapeutic effects of attending group sessions. While these improvements were mainly reported in the intervention groups, even those in the control group reported some benefits. This study highlights that it is both feasible and advantageous to embed qualitative research within the traditional RCT methodology to arrive at a more nuanced understanding of patient experiences and intervention outcomes.

Keywords: Memory rehabilitation; Effectiveness; Qualitative research; Patient perspectives; Neurological disabilities.

Correspondence should be addressed to Roshan das Nair, B09, International House, Institute of Work, Health \& Organisations, The University of Nottingham, Nottingham NG8 1BB. Email: roshan.nair@nottingham.ac.uk 


\section{INTRODUCTION}

Focusing solely on quantitative outcomes to evaluate the effectiveness of neuropsychological rehabilitation offers several challenges for researchers and clinicians. Challenges arise due to issues related to the sensitivity of measures to detect subtle changes, with most assessments being ordinal level measures; the difficulty of ascertaining whether patients have understood questions as intended by the researcher; relying on patient literacy; and the lack of experiential data that may help us make sense of or interpret outcomes. Dingwall (1992) referred to the "black box" of an intervention, which is a useful construct to consider in cognitive rehabilitation, as the processes involved in neuropsychological rehabilitation still remain largely uncharted. He suggested that in evaluating healthcare interventions, structure, process, and outcome be assessed. The usefulness of combined (qualitative plus quantitative) methods in medical evaluation research has been emphasised by Hearn, Lawler, and Dowswell (2003). These authors noted discrepancies between findings from qualitative and quantitative data in a stroke rehabilitation trial, and observed that "the qualitative study provided a useful and different picture from that presented by the quantitative study", and that it "provided a more positive and more encouraging view than was apparent from looking at the quantitative results alone" (p. 33). Therefore, the quantitative outcomes of the RCTs only tell part of the story.

The utility of subjective ratings of outcomes following interventions has been established in several studies. Notwithstanding the problems associated with reporting biases, such measures have found a place in neuropsychological rehabilitation research. Berg, Koning-Haanstra, and Deelman (1991), for instance, elicited participant feedback in terms of subjective ratings on a 10-point scale in their study comparing strategy training versus repetitive practice, in traumatic brain injury (TBI) patients. They found improvements in both experimental and control groups over time on memory, coping, insight, and anxiety. das Nair and Lincoln (2012) used the Everyday Memory Questionnaire as a way to assess intervention effectiveness of two memory rehabilitation programmes compared to a selfhelp control group. "Such measures do not offer a nuanced understanding of the patient's problems articulated in their own words. Qualitative research methods, however, can offer rich descriptions of phenomena and 'enhance understanding of the context of events as well as the events themselves" (Sofaer, 1999, p. 1101). Evans and Wilson (1992) also elicited feedback on their memory group from their participants "informally" throughout the 11 months of their programme. They also used a questionnaire to examine what individuals found helpful, enjoyable, unhelpful, or had disliked about the groups. It is beyond the scope of the present study to unravel the structure and process aspects of the "black box", but some 
information regarding the process can be gleaned by examining the qualitative data reported here.

Feedback interviews serve several functions, and should be considered in RCTs. Firstly, they serve as a debriefing exercise for participants. Debriefing consists of informing trial participants about their individual treatment allocation and study results (Di Blasi, Crawford, Bradley, \& Kleijen, 2005). Debriefing in RCTs is considered good practice, and is also emphasised in government standards for research (Department of Health, 2001); and yet, is overlooked by most trialists (Di Blasi, Kaptchuk, Weinman, \& Kleijnen, 2002). Secondly, they afford participants an opportunity to air their views about the trial itself, and how it can be improved. This is essential in upholding the tradition of patient partnership, which has become a key feature in most NHS services (Department of Health, 1998). Thirdly, they offer qualitative information that cannot be obtained on subjective and objective outcome measures used in most trials.

\section{Aims}

The aims of this study were to elicit and examine feedback from participants who had been through a randomised controlled trial (RCT) comparing two types of memory rehabilitation with a self-help control. It was envisaged that this information would offer a more nuanced understanding of patient experience of going through a trial and the perceived effects of having attended group sessions.

\section{METHODOLOGY}

\section{Epistemological position}

In keeping with the spirit of qualitative methods, a critical realist perspective was taken to understand knowledge production and meaning-making of the participants' feedback. Critical realism assumes that reality can exist independently of human thought. Therefore, all observations and measurements of behaviour are fallible, and truth claims can only ever be an approximation, based on the interpretations of the participants and researchers (see Bhaskar, 1978).

Ethical approval for the study was obtained from Nottingham Research Ethics Committee 1.

\section{Participants}

Participants were recruited from the pilot phase of the ReMIND trial (Rehabilitation of Memory in Neurological Disabilities), a randomised trial of memory rehabilitation (das Nair \& Lincoln, 2012). Individuals were included 
in the trial if they were over the age of 18 , and reported memory problems due to a TBI, stroke or multiple sclerosis (MS); having been diagnosed at least one month prior to recruitment and having no previous diagnosis of brain injury or other severe disability. Participants were excluded if they did not speak English or lived more than 50 miles from Nottingham or Derby (see das Nair, 2007; das Nair \& Lincoln, 2012, for details about the trial).

Thirty-six participants were recruited for the pilot phase of the ReMIND trial, and all were invited to take part in the feedback interviews held at the second follow-up assessment (seven months from randomisation). However, only 31 completed the trial and attended the second follow-up session and interview part of the study.

Therefore interviews were conducted with 24 women and 7 men. All identified themselves as being White British. The majority of them had a diagnosis of MS $(n=21)$, with the remainder having had a stroke $(n=$ 6 ) or TBI $(n=4)$. The mean age of the participants was 45 years ( $S D$ 9.61, range 18-66). They had an average of 13 years of education (SD 2.43, range 8-16) and an average pre-morbid estimated IQ (assessed on the National Adult Reading Test) of 107.11 (SD 9.03, range 86-124). As a group, the overall mean memory scores on the Doors and People Test for the compensation group was 10.4 (SD 9.2), for the restitution group was 6.6 (SD 2.8) and for the self-help group was 6.2 (SD 2.9). The group as a whole did not show evidence of any receptive or expressive language problems as assessed on the Sheffield Screening test for Acquired Language Disorders.

\section{The rehabilitation programme}

Participants in both compensation and restitution programmes were taught the use of internal memory aids and errorless learning techniques. In addition, those in the former group were taught how to use external memory aids. The latter group practised encoding and retrieval strategies (e.g., using the Who, What, Where, When, Why and How questions), which also included attention retraining exercises (e.g., cancellation tasks). The self-help group were not taught any memory strategies, but were taught relaxation techniques and ways in which they could cope with their condition.

\section{Procedure}

The interviewees came from those who were assigned to the "restitution" ( $n$ $=9)$ or 'compensation' $(n=10)$ memory rehabilitation (intervention) groups, and also those in the self-help (control) group $(n=12)$. To reduce social desirability bias, the feedback interviews were conducted by an individual who was not directly involved with the assessments or intervention aspects of the trial. A semi-structured interview schedule was developed 
based on extant literature and in relation to the content of the groups they attended. This interview schedule focused on the following domains: changes to personal, professional and social life, related to mood (e.g., "What effects have you noticed on your personal life as a result of the group sessions?"), cognitive functions (e.g., "What effects have the sessions had on your planning and organisational abilities?"), and strategy use (e.g., "What memory aids or techniques of your own have you developed based on those you were informed about during the sessions?"). It also contained standard prompts (e.g., "That's interesting, can you tell me more about that. . .") to facilitate a conversational style of interaction. The interviews were audio recorded, with the consent of the participant, and transcribed by the interviewer. The transcripts were checked by the first author against the original audio recording, and any errors were corrected.

Transcripts of the feedback obtained using the semi-structured interview schedule were the data corpus to be thematically analysed.

\section{ANALYSIS}

The data were analysed using thematic analysis. The flexibility of thematic analysis and its theoretical freedom, along with the well-established guidelines for performing the analysis, informed the choice of the method. Other methods, such as Interpretative Phenomenological Analysis were also considered, but given some of their theoretical insufficiencies (e.g., Giorgi, 2008) and requirement for small sample sizes (Smith, Flowers, \& Larkin, 2009) which would limit heterogeneity, thematic analysis was considered to be a better method to address the aims of the study. We adopted an exploratory approach and thematic analysis was chosen to unpack meaning and people's experiences and their reality.

In using thematic analysis, we aimed to identify a limited number of themes that adequately reflected the textual data from the interviews. We followed the six phases of thematic analysis proposed by Braun and Clarke (2006). The transcript was read and re-read by both authors which enabled us to familiarise ourselves with the data. The data were coded independently. This enabled us to systematically code the salient points and interesting features of the interview data across the entire dataset. This related to the "generating initial codes" phase. We then searched for themes by collating data relevant to each code. In considering the thematic structure that we would adopt to make sense of the data, we focused on what we felt were the most salient points that participants came back to during the interviews several times without being prompted. This enabled us to review the themes. Finally, we defined and named the themes. 


\section{Quality assurance}

Dual independent coding enabled external validation of the analysis to some extent. Finally, through discussion, we arrived at a consensus regarding the coding and thematic structure, and ensured that we could both "see" the theme in the data, and selected parts of the transcript which exemplified each theme. In ensuring the quality control, we relied on Yardley's characteristics of good qualitative research, by paying close attention to sensitivity of context, commitment and rigour, transparency and coherence, and impact and importance (Yardley, 2000, p. 219). Furthermore, we maintained a research diary to record our own thoughts and feelings associated with the progress of the study and our reactions to our discussions. This also provided us a reflexive and interpretative framework to rely on when analysing the transcript.

Working with a small pool of participants, we were clear from the outset that the aim was not for data saturation, but rather what Dey $(1999$, p. 117) referred to as "theoretical sufficiency". This is another way of assessing the quality and thoroughness of the data analysis without suggesting that the data has been exhausted of themes (see Dey, 1999 for a critique of saturation).

In providing quotes from the data related to each theme, we have in some instances supplied the reader with more than one quote to illustrate the theme more fully. Offering more than one quote allows us also to show "deviant case" examples (we did not observe any other deviant cases apart from those mentioned here). This, we felt, was important to demonstrate transparency and coherence of our analyses and interpretations.

\section{RESULTS}

The major themes that emerged from these interviews were insight and awareness of the participants' illness or condition, and their memory problems; development of knowledge about their neurological condition and memory, and skills to deal with memory problems; improvements in memory function and other cognitive functions; improvements in mood; improvements in confidence, assertiveness and control; an altered perspective of life; and the therapeutic effects of being in a group setting. These themes are explored below, with excerpts from the transcripts which highlight the participants' experiences. Rather than assigning pseudonyms, we only report the gender (M/F), diagnosis (TBI/MS/Stroke), and the group the participant was allocated to (restitution/compensation/self-help). This was done to protect the identity of the participants, but at the same time providing some relevant information for the reader to contextualise the quote. 


\section{Insight and awareness}

The importance of improving patients' awareness of neuropsychological deficits to help them use compensatory strategies has been stressed by Klonoff et al. (1989) and Prigatano (1999). This helps them make appropriate and suitable decisions and life choices (Prigatano, 1999). In this study, some participants reported that they understood their neurological condition and the memory problems better because of the group sessions.

"Yeah, like I say, because all four of us were discussing our symptoms it made you feel... understand the symptoms a little bit more." (F, MS, Self-help)

The sessions permitted participants to "own" their memory problems, not feel ashamed of them, and feel comfortable talking about them. This awareness and understanding was important because it permitted participants to find ways to address their problems, or learn to live with them.

“... having that acknowledgement and permission from [researcher] to say it was all right, it can be happening because of your MS, and just having someone to acknowledge that, was like a huge weight off my shoulder. Because I felt like I was either going insane or I was maybe being a bit of a hypochondriac... but now I can deal with it [memory problem]." (F, MS, Compensation)

“. . . it's [attending groups] made me more open, when I get stuck with words, I just say, 'I'm very sorry, my brain's not functioning', whereas before I'd just get very. . .umm... frustrated." (F, MS, Compensation)

All groups had a session on the theoretical aspects of their neurological condition and memory. The reactions to this session was mixed, but was largely positive. Some did not benefit from it:

“... because I think that was more technical. And my brain didn't absorb it all to be honest." (F, MS, Compensation).

However, most participants from all three programmes reported benefits from this theoretical session.

"It was useful. When he [researcher] started going into it. I was like...

'Oh gosh, you know, do we really need to go that deep? Can't you just 
tell me how to manage it?' But as you go through the sessions, it all clicked into place. So I think it was needed." (F, MS, Compensation)

“... it really make[s] sense because sometimes now I tend to visualise what's happening in my brain. And if it's actually gone somewhere and stored. Or whether it's gone here and gone straight out. . . It actually is amazing to sort of visualise what your brain's doing 'cos you don't think about it until somebody points out to you that your brain's actually like a great huge filing system. And that was actually quite interesting." (F, MS, Self-help)

\section{Knowledge and skills}

The sessions provided participants with strategies which they could use to address their memory problems (for the treatment groups) or stress (for the self-help groups). Giving the strategies names (e.g., "chunking", "imagery", etc.) was found to be beneficial.

“. . . now that I can actually think which one [strategy] shall I use, which is the best one to use, because I've been given names as such. So it's easier for me now to pull in a particular strategy... sort of a tool from my toolbox to use which is appropriate for me for that particular task. So yeah, I'd say I'm a little more maybe organised." (F, MS, Compensation)

Many participants in the compensation groups began using a range of external memory aids, most notably diaries, notebooks, post-its, noticeboards, mobile phones and calendars. Participants were not only provided with such a "toolbox", but were also encouraged to have a positive attitude towards using the tools. One lady commented,

"They [husband and daughter] laugh at me, both of them, when they see my Post-its in the car, the Post-its in the kitchen... and I say, 'but it's all right for you, but I need this'. I need to have the reminders." (F, MS, Compensation)

The sessions provided an opportunity for those who were already using external memory aids to examine the effectiveness of these aids, and to consider other alternatives which were perhaps more suitable for them.

"So the session allowed me to sort of instead write... rather than have Post-its everywhere I decided to keep a small book and log each item or thing that I needed to do. .. then I ended up with having 53 items at one 
point. But I was crossing them off as I was doing them. So I didn't need to have a disorganised. . . Post-its everywhere. And that was through the session really. It was just [a] simple thing, it was just to use a book, really." (F, MS, Compensation)

Some participants learnt to use external memory aids themselves, and then implored others in their environment to follow suit. One lady had a "family diary" which she placed in the kitchen. The family were instructed to write in it if they required her to do something for them. Her motto was simple, "If it's not in the diary, you haven't told me!". Another participant used a calendar to reduce forgetting and stress.

"At home we use a calendar where we put all our [activities in]... what I'm doing, what my husband's doing, what my son's doing... and that was a bit half-heartedly being done but the session's sort of shown me that it's a good thing to have... because it does prevent arguments and unnecessary stress in the house." (F, MS, Compensation)

Participants in the restitution groups began to use mental strategies to reduce forgetting. An improvement in their ability to pay attention to the task at hand and a general sense of being aware of what they were doing perhaps helped.

"I am able to now... like I said... to sort of think, 'right, stop, you've forgotten something and just take that moment to remember', so... whereas before I never did. I used to just go... 'ugh... forgot!"” (F, MS, Restitution)

"... being more aware of what is going on around me as well, where I have parked [the car] and concentrate more as well." (F, MS, Restitution)

Participants took on board the importance of errorless learning, by paying more attention to the information being learnt, not guessing, and going though procedures step-by-step.

"I've also learned to do. .. I should never just think I can do something without reading the instructions because I always used to do that. Whereas now I read the instructions because... if you make a mistake when you do it first time it stays in your head. So I don't do that now." (F, MS, Compensation) 
This theme was endorsed by those in both the intervention groups, but not the self-help group.

\section{Improvements in cognitive functions}

Where improvements in cognitive functions were reported, they were mainly in attention, memory, planning, and problem solving. Again, gains were not restricted to the compensation groups, but participants in the restitution groups also reported improvements.

“... I'm a better planner... I do plan things better. I definitely do this with my lists, my Post-its and things so I... I am better organised. I'm not saying I don't forget things. I still do forget things. But I am better. Now I know I need to. And that makes a difference... it's taught me that I need to pay more attention really to what I'm doing." (F, MS, Compensation)

"My husband... seems to know where everything in the house is... It makes me slightly nervous, I can feel myself almost thinking at one point Anthony will know where it is. 'Anthony can you find this?' Now I think I don't want him to be doing it, I should know where it is. If I want the clippers for the garden then I should know where they are. 'Think where would they be when you came in?' Then I should find them." (F, MS, Restitution)

Interestingly, despite providing no specific intervention to improve cognitive functions, participants in the self-help group also seemed to show signs of progress, perhaps mainly though an improvement in self-confidence.

“... I plan things better now. Well, I went to find work, part-time work. I was planning to work part-time for quite a while. Up until Christmas I would never have done it any other way. I went out confident with that." (M, TBI, Self-help)

\section{Improvements in mood}

In all three groups, most participants reported an improvement in their mood. This manifested as reduced stress, frustration, worry, and feeling more relaxed, positive, and feeling and looking better.

"I don't get as worried when I forget things that I used to. And before if I forgot something it really upset me but now I think 'No', I know why I forgot it, so I take a moment to myself and then I'm able to more often 
than not remember now. . . and I have got different ways now of remembering things." (F, MS, Compensation)

"I'm a lot more relaxed. I could worry for England, or used to be able to worry for England. I probably still do, I don't know. But I do feel as though I'm a little more relaxed." (M, Stroke, Self-help)

"Definitely improved [mood], certainly at home. I'm a lot, because I feel a lot more comfortable with my memory thing because I'm not having constantly to ask to remember, and I'm not forgetting things and that was the most frustrating part that I was just forgetting I had to be somewhere or go, you know. .." (F, MS, Compensation)

"I notice I have been more positive, I sort of feel better. You know people will say to me, 'You look better', whether it's just me, I don't know. But people have noticed that I do look better in myself." (F, Stroke, Restitution)

However, not everyone reported benefits in terms of mood.

"I wouldn't say that I noticed any difference in my mood as a result of the classes. I think for me in my personal circumstances the classes were a bit too late... I had already looked up and had tried a lot of different learning methods.. ." (F, TBI, Restitution)

\section{Confidence, assertiveness, and control}

Most participants, in all three groups, reported feeling more confident as a result of the sessions. The sessions appeared to have provided participants with a feeling of control to their lives, and most reported being able to assert themselves better in social situations. Participants appeared to have developed a sense of competence as a result of knowing that something could be done to reduce forgetting, and knowing what to do.

“. . . I tell you, the biggest thing is confidence. Because it really was upsetting me and I really did think it was worse than probably what my memory is really. My husband can't get away with fibbing any more. . ." (F, MS, Compensation)

"I feel better because I know now how to relax more. If I get stressed, I know how to... how I can control the stress." (M, Stroke, Self-help) 
“. . . I have started to do other things that I haven't done before, because I have got more confidence... ahhh... and I have got more confidence that I can remember things a bit easier than I used to." (F, MS, Restitution)

"I'm even able to say, 'Excuse me what was that you said there? What was that dear? I can write it down.' I'm not afraid to speak and ask again. Because before I was kind of thinking you're mad if you can't remember that bit. It's just that whole anxiety playing up. So now I can more assertive.” (F, MS, Restitution)

\section{Altered perspective of life}

For some participants, the sessions made them think about life differently. Whether it was from a practical manner of doing things differently or from adopting a stoical position, this transformation was observed across all three groups. However, this was most notable in the self-help groups where participants had an opportunity to discuss and reflect on what made their lives difficult, and how this could be changed.

"[I] take a step back now and look at things rather than sort of, diving in, and I've also learned to say 'No' quite a lot. It is. . . for me because I used to say 'Yes' to everything and found myself disappearing on my own backside... disappearing, just going round and round in circles and getting nowhere, so that was good, it did help me." (M, Stroke, Self-help)

"What it has helped me to do is to be more organised in the things that I set myself. And again this makes me feel better about me... Now I am disciplined and I make a list and I don't deviate from that list... I feel so good... might be a very small bit of gardening but I have achieved something." (F, MS, Compensation)

“. . . the one thing is it taught me just how to switch off, say, 'Bugger it!'” (M, Stroke, Self-help)

"It has made me think, 'Well perhaps some things you cannot change and you have got to live with it and some things you can change and you have got to work at it."” (F, Stroke, Self-help)

"I do see life in a different way now. I can go out in the street knowing that it's just... 'It's only a blip, get on with it."' (M, TBI, Self-help) 
“. . . after [the] brain injury I was so irate and quite short-tempered. And I came out of the meeting thinking I know how to calm down and settle down and so I can get on with it. I approach things differently now." (M, TBI, Self-help)

“... so that the sessions did actually teach me to say 'Stop, step back a little bit' and not putting too much expectation on myself, little steps, little bits at a time." (M, Stroke, Self-help)

\section{Therapeutic effects of the group}

The therapeutic effects of the attending support groups has been documented in various conditions, such as cancer (Goodwin et al., 2001), and MS (Holmes, Ford, Yuill, Drummond, \& Lincoln, 2012; Lincoln et al., 2011); and was endorsed by all three groups in our study. Research in ageing has indicated that psychological and social factors, including lifestyle-related activities have an interaction effect that may reduce adverse effects of brain dysfunction on cognition (e.g., Dawson, Winocur, \& Moscovitch, 1999). Such mediating effects may also play a role in recovery from patients' neurological condition, and therefore need to be acknowledged in cognitive rehabilitation programmes and evaluation of their outcomes. While such support from the groups in themselves may not have directly improved memory, participants found it useful for a number of reasons. For some, the groups served as a social event.

"It gave me somewhere to go. I did enjoy coming to the sessions. I did enjoy meeting one or two people, one of whom I am still in contact with." (F, TBI, Restitution)

For others they provided a sense of community, and an arena to vent their frustrations, exchange ideas, and to learn.

"[You] sit in a group, say something, and the other people in the group will say back to you... 'Oh I get that'. It was quite nice. I suppose it just puts your confidence up that you're not the only person that does braindead sort of things." (F, MS, Restitution)

"I think the biggest thing for me was listening to other people and realising that I'm not alone and I could laugh at a lot of things rather than becoming very anxious about it. With the sharing of... so it's taken away a lot of the anxiety around things that are not because of the anxiety, and laughing at what's going on." (F, MS, Self-help) 
"So as a group we've all managed to come up with lots of new ideas." (F, MS, Compensation)

The group also afforded individuals an opportunity to examine their own experiences, to process what was being discussed and to reflect on issues.

“... [Being in a group] takes the pressure off a bit if there are two or three people... because you can take any piece of information and could process that information whilst someone is talking. So you don't feel that you've got to constantly be responding you can take that quiet time for yourself to think things over in your brain." (F, MS, Compensation)

While the heterogeneity of the group was considered a potential problem in the study, many participants did not view this as a hindrance to their level of understanding each other and sharing concerns.

“... with different age ranges, with different lifestyles and whatever, but we all have the same problem. It was quite a comforting feeling and also I think we all sort of said it we could talk to each other and say what sort of problems we got and not feel silly doing it because we'd all got... or at some point in time, we'd all had the same problem." (F, MS, Self-help)

The small size of the group permitted people to feel comfortable to share their experiences. One participant spoke of his experience of a previous programme with larger groups.

"Whereas the group that I went in before was a big group of about 8 to 9 people it was all too much for me." (M, Stroke, Self-help)

\section{DISCUSSION}

From the transcripts of the feedback interviews it is clear that changes occurred and were reported in some instances but not in others. When changes were reported, they tended to be in various domains and to varying degrees. Some participants reported more insight and awareness of their illness or condition, and their memory problems. This permitted some to continue using the memory aids they were used to more consistently and effectively, or experiment with other memory aids as replacements or adjuncts to them. Some participants reported improvements in memory and other cognitive functions, mainly attention, planning, and problem solving. This, however, was mostly reported among participants in the intervention 
groups. Improvements in mood and feeling more confident, assertive, and in control, were reported by some participants irrespective of the group they attended. Many participants in the self-help group reported having an altered perspective of life, which is not surprising as a considerable amount of time in this group was spent discussing feelings, illness-related issues and learning how to deal with stress. These discussions may have facilitated some lifestyle changes at a cognitive, emotional, and behavioural level. All but one participant reported having enjoyed working in a small group, and almost all participants felt they had benefited from attending the groups, irrespective of the group they had been allocated to.

Cognitive rehabilitation is a complex intervention. Complex interventions are those that include several components with various interconnecting parts (Campbell et al., 2000). In such interventions, it is difficult to define with precision the active ingredients, and how these relate to each other (Hawe et al., 2004). While some interesting themes emerged from the thematic analysis carried out on the feedback interviews, it was beyond the scope of this study to examine these ingredients in greater detail. These need to be examined in larger studies using in-depth qualitative methodologies.

Some of the results obtained from the quantitative data from the RCT (das Nair, 2007; das Nair \& Lincoln, 2012) and the qualitative data from the feedback interviews were discrepant. Such divergent findings when employing two methodologies are not uncommon (Moffatt, White, Mackintosh, \& Howel, 2006), and may be indicative of different aspects of the phenomena being investigated. Other studies have also reported this discrepancy between findings from feedback interviews and Rivermead Behavioural Memory Test (RBMT) scores. Evans and Wilson (1992) reported positive feedback, a significant increase in the overall use of memory aids and strategies, and some improvements in mood, but no significant changes on the RBMT. The authors acknowledged that as theirs was an uncontrolled study, no firm conclusions could be drawn from these findings. Furthermore, data were only reported for five participants (with complete data for only four). The authors suggested that the lack of change observed in memory functioning (as measured by the RBMT) supported the view that memory rehabilitation groups should help patients make better use of memory aids and strategies and not attempt to improve memory functions themselves (Evans \& Wilson, 1992). Tam and Man (2004) also observed no significant differences on RBMT, in their memory rehabilitation study, although clinical improvements were seen postintervention. Quemada et al. (2003) have also reported finding no change on RBMT scores post-treatment, in their memory rehabilitation study with patients with TBI, when functional gains were observed anecdotally. The lack of changes on these measures, when changes on subjective measures were reported, is perhaps due to the scaling of the RBMT and the RBMT-E, which reduces its sensitivity in picking up treatment-induced 
changes. Furthermore, effective techniques applied post-intervention, such as behavioural adaptation and situation-specific procedural learning, were unlikely to significantly affect performance on name and face recall or remembering appointments, which are subtests on the RBMT (Quemada et al., 2003). Similarly, modest sample sizes and lack of sensitivity of some outcome measures may have contributed to the lack of measurable differences between groups in the study by das Nair and Lincoln (2012).

One limitation of this study is that only the first 31 participants were recruited for the interviews, i.e., only those in the pilot phase of the RCT, and like most other qualitative studies, only offers a snapshot of a few peoples' experiences. All those who completed the pilot trial took part in the interview. Those who dropped out before their follow-up assessments may have had less positive experiences, and these experiences were therefore not recorded. We did not complete a sub-group analysis of the qualitative data on the basis of clinical diagnosis. This was consonant with our reporting of the quantitative findings from our RCT. However, we acknowledge that there may have been diagnosis-specific experiences that were overlooked because of our decision to analyse the sample as a whole. The interviewer, although independent, had been involved in memory rehabilitation studies, and therefore may have been biased towards prompting responses that elicited the benefits of the intervention. Also the quality of evidence rests on the skill of the interviewer. Although our interviewer was trained by the study authors who have experience in conducting interviews for qualitative research, there were instances where the interviewer asked closed questions and which may have affected the quality of the data collected. The interview style and questions posed, however, were taken into consideration when interpreting the data. We also acknowledge the potential for bias, in that we conducted the RCT and were therefore familiar with what each of the RCT group interventions was aiming to achieve and the mechanisms by which they hoped to achieve them. However, it is hoped that such bias was minimised by our rigour in analysing and reporting the data (as mentioned above).

Despite these limitations the information complements the quantitative analysis and highlights the strengths and limitations of these on the effectiveness of memory rehabilitation. The study itself also highlights the feasibility and usefulness of complementing traditional RCT methodologies with qualitative data, which may help triangulate research findings and offer a more nuanced interpretation of some of the quantitative findings.

\section{REFERENCES}

Berg, I. J., Koning-Haanstra, M., \& Deelman, B. G. (1991). Long-term effect of memory rehabilitation: A controlled study. Neuropsychological Rehabilitation, 1, 97-111. 
Bhaskar, R. (1978). A realist theory of science. Brighton: Harvester Press.

Braun, V., \& Clarke, V. (2006). Using thematic analysis in psychology. Qualitative Research in Psychology, 3, 77-101.

Campbell, M., Fitzpatrick, R., Haines, A., Kinmonth, A. L., Sandercock, P., Spiegelhalter, D., \& Tyrer, P. (2000). Framework for design and evaluation of complex interventions to improve health. British Medical Journal, 231, 694-696.

das Nair, R. (2007). Effectiveness of memory rehabilitation following brain damage: A single blind randomised controlled trial. $\mathrm{PhD}$ Thesis submitted to the University of Nottingham.

das Nair, R., \& Lincoln, N. B. (2012). Evaluation of memory rehabilitation in people with neurological disabilities: A randomised controlled trial (REMIND). Clinical Rehabilitation. doi: 10.1177 / 0269215511435424

Dawson, D., Winocur, G., \& Moscovitch, M. (1999). The psychosocial environment and cognitive rehabilitation in the elderly. In D. T. Stuss \& G. Winocur (Eds.), Cognitive neuroreh-abilitation (pp. 94-108). Cambridge: Cambridge University Press.

Department of Health (1998). A first class service: Quality in the new NHS. Wetherby: Department of Health.

Department of Health (2001). Research governance framework for health and social care. Available from http://www.dh.gov.uk/assetRoot/04/10/89/65/04108965.pdf. [last accessed 1 May 2007].

Dey, I. (1999). Grounding grounded theory: Guidelines for qualitative inquiry. San Diego, CA: Academic Press.

Di Blasi, Z., Crawford, F., Bradley, C., \& Kleijnen, J. (2005). Reactions to treatment debriefing among the participants of a placebo-controlled trial. BMC Health Service Research, 5, 30.

Di Blasi, Z., Kaptchuk, T., Weinman, J., \& Kleijnen, J. (2002). Informing participants of allocation to placebo at trial closure: Postal survey. British Medical Journal, 325, 1329.

Dingwall, R. (1992). "Don't mind him - he's from Barcelona": Qualitative methods in health studies. In J. Daly, I. McDonald, \& E. Willis (Eds.), Researching health care: Designs, dilemmas, disciplines. London: Tavistock/Routledge.

Evans, J. J., \& Wilson, B. A. (1992). A memory group for individuals with brain injury. Clinical Rehabilitation, 6(1), 75-81.

Giorgi, A. (2008). Concerning a serious misunderstanding of the essence of the phenomenological method in psychology. Journal of Phenomenological Psychology, 39, 33-58.

Goodwin, P., Leszcz, M., Ennis, M., Koopmans, J., Vincent, L., Guther, H., et al. (2001). The effect of group psychosocial support on survival in metastatic breast cancer. New England Journal of Medicine, 345, 1719-1726.

Hawe, P., Webster, C., \& Shiell, A. (2004). A glossary of terms to navigate the field of social network analysis. Journal of Epidemiology \& Community Health, 58, 971-975.

Hearn, J., Lawler, J., \& Dowswell, G. (2003). Qualitative evaluations, combined methods and key challenges: General lessons from the qualitative evaluation of community intervention in stroke rehabilitation. Evaluation, 9, 30-54.

Holmes, J. M., Ford, E., Yuill, F., Drummond, A. E. R., \& Lincoln, N. B. (2012). Attendance at a psychological support group for people with multiple sclerosis. Disability and Rehabilitation, 1-5. doi:10.3109/09638288.2011.642927

Klonoff, P. S., O’Brien, K. P., Prigatano, G. P., Chiapello, D. A., \& Cunningham, M. (1989). Cognitive retaining after traumatic brain injury and its role in facilitating awareness. Journal of Head Trauma Rehabilitation, 4(3), 37-45.

Lincoln, N. B., Yuill, F., Holmes, J., Drummond, A. E. R., Constantinescu, C. S., Armstrong, S., \& Phillips, C. (2011). Evaluation of an adjustment group for people with multiple sclerosis and low mood: A randomised controlled trial. Multiple Sclerosis, 17, 1250-1257. 
Moffatt, S., White, M., Mackintosh, J., \& Howel, D. (2006). Using quantitative and qualitative data in health services research - what happens when mixed method findings conflict? BMC Health Service Research, 6, 8.

Prigatano, G. P. (1999). Commentary: Beyond statistics and research design. Journal of Head Trauma Rehabilitation, 14(3), 308-311.

Quemada, J. I., Munoz, C., Juan, M., Ezkerra, J., Ballesteros, J., Ibarra, N., \& Urruticoechea, I. (2003). Outcome of memory rehabilitation in traumatic brain injury assessed by neuropsychological tests and questionnaires. Journal of Head Trauma Rehabilitation: Focus on Clinical Research and Practice, Part 3, 18(6), 532-540.

Smith, J. A., Flowers, P., \& Larkin, M. (2009). Interpretative phenomenological analysis: Theory, method and research. London: Sage.

Sofaer, S. (1999). Qualitative methods: What are they and why use them? Health Services Research, 34(5), 1101-1118.

Tam, S.-F., \& Man, W.-K. (2004). Evaluating computer-assisted memory retraining programmes for people with post-head injury amnesia. Brain Injury, 18(5), 461-470. Yardley,

L. (2000). Dilemmas in qualitative health research. Psychology \& Health, 15(2), 215-228. 